\title{
Wave Equations and Solutions of In Vacuo and Fluid-Filled Elliptical Cylindrical Shells
}

\author{
Abhijit Sarkar and Venkata R. Sonti \\ Facility for Research in Technical Acoustics, \\ Department of Mechanical Engineering, \\ Indian Institute of Science, Bangalore - 560 012, India.
}

(Received 10 May 2008; accepted 20 January 2008)

\begin{abstract}
A perturbation-based approach is used to formulate the governing equations for wave propagation at high frequencies in infinite in vacuo and fluid-filled elliptical cylindrical shells. The in vacuo equations thus formulated have the form of a perturbation over the corresponding equations for the circular cylindrical shell. Here, eccentricity of the cross-section is assumed to be small and used as the perturbation parameter. Next, the coupled equations for the fluid-filled elliptical shell are obtained as a perturbation over the in vacuo shell equations (by using a single fluid-loading parameter $\mu$ ). Asymptotic arguments are used to neglect various terms in the derivation, and a compact form of the non-dimensional governing equations is found. Presenting the governing equations in this form is the main contribution of this work. Due to the eccentricity, all spatial quantities need to be represented in terms of a harmonic series instead of a single harmonic term. Using symmetry and asymptotic arguments, the nature of the harmonic series is obtained. Using the harmonic series expansion, the dispersion relation is formulated for both the in vacuo and the fluid-filled cases. The in vacuo dispersion equation is solved using the regular perturbation method, while the transcendental coupled dispersion equation is solved numerically.
\end{abstract}

\section{INTRODUCTION}

The coupled wavenumber characteristics of a fluid-filled flexible infinite shell have been a major area of study in acoustics. The fluid and the shell form a coupled structural acoustic waveguide where the wave propagation in one medium is modified by the influence of the other. The importance of these studies lies in understanding values of system parameters where the shell and the fluid can be considered as separate (or independent of one another), and also those where the shell and the fluid are well coupled and need to be considered as one system. Earlier work in this direction has been mainly limited to the circular cylindrical geometry. ${ }^{1-3}$ Another regular geometry worth considering is the elliptical cylindrical system, which poses greater mathematical challenges specially if one uses analytical methods. This is evidenced by the sparsity of published literature.

In the literature, there have been a few isolated analytical studies on vibration and acoustics of the elliptical cylindrical geometry. Lowson and Bhaskaran studied the acoustic wave propagation in rigid-walled elliptical ducts. ${ }^{4}$ They formulated the dispersion relation and compared the cut-on frequency characteristics to the case of circular cylindrical ducts. Similar to their work, Hong and $\mathrm{Kim}^{5}$ found the natural frequencies and mode shapes of finite length hollow and annular elliptical cavities with rigid-walled boundaries. They also discussed the transition of the results to the circular cylindrical geometry for the limiting case of zero eccentricity. Willatzen and Voon ${ }^{6}$ included the effect of bulk flow properties in rigidwalled elliptical cylindrical geometries for both infinite waveguides and finite enclosures. They showed that for any acoustic mode, the phase speed is increased by the flow velocity along the direction of the wave propagation.

For the in vacuo structural dynamics, Leissa $^{7}$ reported the use of a Galerkin method for finding the natural frequencies of an in vacuo elliptical cylindrical shell. Soedel ${ }^{8}$ used the Galerkin method to study the in vacuo structural dynamic parameters of shells with arbitrary geometries. Natural frequencies for finite length elliptical shells have been evaluated using the Rayleigh-Ritz method for various boundary conditions, ${ }^{9-11}$ where the trigonometric functions ( $\sin$ and $\cos$ ) were used as shape functions. Shirakawa and Morita ${ }^{12}$ studied the vibration of elliptical shells by considering the elliptical cross-section to be composed of two circular arcs. This allowed them to use the simpler circular cylindrical shell equations.

Though using FEM-BEM-based numerical approaches, the dynamic response of the fluid-filled elliptical shell system can be found; an analytical form of the governing equations for this system has not come to our notice. In this article, we formulate the governing equations for the in vacuo and the fluid-filled elliptical cylindrical shells. Eccentricity and the fluid-loading parameter ( $\mu$, defined later) will be used as the asymptotic parameters. Finally, the equations will take the form of the circular cylindrical shell equations with a perturbation due to eccentricity (leading to the in vacuo elliptical cylindrical shell) and another perturbation from the fluid-loading parameter (leading to the fluid-filled elliptical cylindrical shell).

\section{THE ELLIPTICAL COORDINATE SYSTEM}

The elliptical coordinate system is shown in Fig. (1b). The coordinates are parameterized by $\xi, \eta$, and $z$, where $\xi, z \in$ $(0, \infty)$ and $\eta \in(0,2 \pi)$. The $\xi=$ constant curves comprise a family of confocal ellipses with the focii at $( \pm f, 0)$, and $\eta=$ constant curves are the orthogonal hyperbolas. $\mathbf{e}_{\xi}, \mathbf{e}_{\eta}, \mathbf{e}_{\mathbf{z}}$ are the unit vectors in the $\xi, \eta$, and $z$ directions, respectively.

The transformation from the $\xi \eta$ to the cartesian coordinates 